\title{
Comparison of Early Outcome of Off-pump and Conventional Coronary Artery Bypass Graft Surgery in Patients with Multivessel Coronary Artery Disease and Left Ventricular Dysfunction
}

Sanjay Kumar Raha1, Md. Sorower Hossain², Smriti Kana Biswas³, Salahuddin Rahaman ${ }^{4}$, Manzil Ahmad ${ }^{5}$, Md. Kamrul Hasan 6

\begin{abstract}
:
Introduction: Left ventricular dysfunction is an important predictor of in-hospital mortality. Due to the theoretical and practical advantages to avoid the harmful effects of cardiopulmonary bypass (CPB), many cardiac surgeons are using Off-pump Coronary Artery Bypass (OPCAB)as an effective alternative to conventional CABG (CCAB) even in patients with reduced left ventricular (LV)ejection fraction.

Objectives: This study performed in the National Institute of Cardiovascular Diseases (NICVD) evaluated the early outcomes of OPCAB in terms of mortality and major post-operative morbidities and compared them with that of CCAB in patients with multivessel coronary artery diseases and reduced left ventricular (LV) function.

Methods: Total 120 patients with multivessel coronary artery disease with reduced left ventricular ejection fraction (d"50\%)were allocated into two groups: a) 60 patients who underwent OPCAB and b) another 60 patients who underwent conventional CABG between January 2013 and December 2015. Pre-operative, peroperative and early post-operative variables were recorded, compiled and compared.
\end{abstract}

Results: All risk factors and co-morbidities were homogenously distributed between the two groups. Majority of the patients had triple vessel disease. Nearly three-quarter $(73.3 \%)$ of patients in OPCAB group and $80 \%$ in CCAB group received 3 grafts $(p=0.470)$. The mean total operative time $(268.5 \pm 33.5 v s .296 .3 \pm 34.8$ minutes, $p<0.001)$, intubation times $(8.6 \pm 0.3$ vs. $12.3 \pm 0.5$ hours, $p<0.001)$, blood losses (377.8378 $\pm 45 \mathrm{ml}$ vs. $602 \pm 60 \mathrm{ml}$, p < 0.001); requirements for blood and blood products $(689.7 \pm 21.1$ vs. $1199.3 \pm 34.5 \mathrm{ml}, \mathrm{p}<0.0010)$, intensive care unit stays $(31.7 \pm 0.9$ hours versus $41.6 \pm 1.5$ hours; $\mathrm{p}<0.001)$ and hospital stays $(8.2 \pm 0.2$ days vs.10.3 \pm 0.3 days, $p<0.001$ )were all significantly lower in the OPCAB group.

Conclusion: OPCAB is a safe and effective operative revascularization procedure for patients with multivessel coronary artery disease and left ventricular dysfunction and is associated with reduced morbidity. However, a larger and omized trial with long-term followup may show the real benefits of OPCAB.

Key Words: OPCAB, CCAB, Multivessel Coronary Artery Disease, Left Ventricular Dysfunction.

(Bangladesh Heart Journal 2020; 35(1): 20-27)

1. Associate Professor, Department of Cardiac Surgery, National Institute of Cardiovascular Disease, Dhaka, Bangladesh.

2. Assistant Registrar, Department of Cardiac Surgery, National Institute of Cardiovascular Disease, Dhaka, Bangladesh.

3. Assistant Registrar, Surgery Outpatient Department, National Institute of Cardiovascular Disease, Dhaka, Bangladesh.

4. Assistant Registrar, Department of Cardiac Surgery, National Institute of Cardiovascular Disease, Dhaka, Bangladesh.

5. Associate Professor, Department of Cardiac Surgery, National Institute of Cardiovascular Disease, Dhaka, Bangladesh.

6. Professor, Department of Cardiac Surgery, National Institute of Cardiovascular Disease, Dhaka, Bangladesh.

Address of Correspondence: Dr. Sanjay Kumar RahaSanjay Kumar Raha, Associate Professor, Dept. of Cardiac Surgery, National Institute of Cardiovascular Disease (NICVD), Dhaka, Bangladesh. E-mail: drsanjayraha77@yahoo.com, Mobile: +88 01720988629

DOI: https://doi.org/10.3329/bhj.v35i1.49139

Copyright $\odot 2017$ Bangladesh Cardiac Society. Published by Bangladesh Cardiac Society. This is an Open Access articles published under the Creative Commons Attribution-NonCommercial 4.0 International License (CC BY-NC). This license permits use, distribution and reproduction in any medium, provided the original work is properly cited and is not used for commercial purposes. 


\section{Introduction:}

Jones et al ${ }^{1}$ examined seven large datasets, with more than 172,000 patients undergoing isolated CABG, to assess the predictive power of certain preoperative values on operative mortality. Seven variables were found to be predictive of mortality in all datasets. These seven core variables included urgency of operation, age, prior heart surgery, gender, left ventricular ejection fraction (LVEF), and percentage of stenosis of the left main coronary artery and number of major coronary arteries with $70 \%$ stenosis. ${ }^{1}$

CABG with cardioplegia has been considered the gold standard operation for coronary revascularization. The introduction of the heart-lung machine created optimal operative conditions of bloodless, motionless operative field and allowed CABG to be done consistently with generally good results in most patients. ${ }^{2}$ However, this technique is linked to several side effects mostly due to use of aortic cross clamp, cardioplegia, and cardiopulmonary bypass. High risk patients are extremely sensitive to cardioplegic arrest and have higher intraoperative and post-operative risk. ${ }^{3}$

Atrial fibrillation occurs in $20-40 \%$ of patients who had CABG with cardiopulmonary bypass and is associated with higher risk of cerebrovascular accidents. ${ }^{4}$ The higher incidence of post-operative IABP insertion, renal hemodialysis, mechanical ventilation and/or reintubation or tracheostomy could be responsible for the significant higher rate of readmission for CPB patients. ${ }^{5}$

The invention of Off-pump Coronary Artery Bypass (OPCAB) is often attributed to Kolessov in 1967. ${ }^{6}$ In the mid-1990s interest in beating-heart techniques experienced resurgence in an attempt to decrease the morbidity associated with CABG without jeopardizing the benefits ${ }^{6}$. Although the initial experience with OPCAB was limited to single or double vessel disease with preserved left ventricular function, ${ }^{7,8}$ the availability of modern retractor-stabilizers, heart positioning devices, techniques of exposure of all surfaces of heart, intracoronary shunts, and adequate surgeon experience, similar completeness of revascularization and graft patency can be achieved with OPCAB even in patients with multivessel disease and reduced left ventricular function. ${ }^{9,10,11}$ Now OPCAB is widely accepted and considered to be safe for myocardial revascularization specially for high risk patients. ${ }^{12}$

The rapidly increasing incidence of diabetes mellitus, hypertension, and hypercholesterolemia in most communities has given rise to more severe and diffuse coronary artery disease. ${ }^{13} \mathrm{As}$ a result of improvement in invasive cardiology most patients referred for CABG have diffuse disease and poor ventricles. The global ischemia caused by conventional CABG (CCAB) could be detrimental to them. The OPCAB technique was developed with specific purpose of reducing mortality and morbidity in high risk patients. ${ }^{6}$

The shorter operating time in OPCAB group is probably because of the shorter time required for hemostasis and no time spent on cannulation, managing cardiac arrest and rewarming from hypothermia during CPB. There is also less intra-operative and post-operative blood loss, a lower incidence of re-exploration for bleeding, a smaller transfusion volume and lower incidence of transfusion. ${ }^{14}$

The shorter ICU stay and ventilation time in the OPCAB group may be related to lack of the pulmonary dysfunction that occurs after CPB. Glomerular filtration rate and renal tubular function are better protected by OPCAB group than by conventional CABG. ${ }^{14}$

There are also potential economic benefits including reduced postoperative patient instability and faster recovery rate, reduced use of vasoactive drugs, less need for blood products and cost effective resource utilization. ${ }^{15}$

\section{Materials and Methods:}

This was prospective non-randomized clinical study was done in National Institute of Cardiovascular Diseases (NICVD) from January 2013 and December 2015. Among the 120 patients with multivessel coronary artery disease with reduced left ventricular ejection fraction (d"50\%) 60 patients underwent OPCAB (Group: A) and the rest 60 underwent conventional CABG (Group: B).

\section{Anesthesia and Monitoring}

Patients were premedicated with morphine $(0.1 \mathrm{mg} / \mathrm{kg})$ and bromazepam ( $3 \mathrm{mg})$. Induction and maintenance was done according to local protocol. All patients had a radial artery cannula, a central venous catheter, ECG leads and nasopharyngeal temperature probe for monitoring. Femoral artery cannulation was performed in patients with poor ventricular function (left ventricular ejection fraction $<35 \%$ ) in the event that urgent institution of an intra-aortic balloon pump was required. Arterial blood gases and activated clotting time (ACT) were monitored every 30 minutes. The patient's temperature was maintained close to $36^{\circ} \mathrm{C}$ for OPCAB and $32-34^{\circ} \mathrm{C}$ for CCAB.

\section{Technique of OPCAB}

All patients were operated through a median sternotomy. The internal thoracic artery, the radial artery, and the saphenous vein were harvested as appropriate with 
standard techniques. Then intravenous heparin (100IU/ $\mathrm{kg}$ ) was given to maintain an ACT of 300 second before starting distal anastomoses. Regional myocardial immobilization was achieved with a suction stabilizer (Octopus) and apical suction device (Star Fish). Intracoronary shunts were used in most patients to maintain coronary flow, thereby reducing myocardial ischemia and at the sametime minimizing bleeding from the coronary arteriotomy. The left anterior descending (LAD) artery was revascularized first using left internal mammary/ thoracic artery (LIMA/ LITA). Proximal anastomoses were performed on the partially clamped ascending aorta using 6-0 continuous Prolene suture. Distal anastomoses were performed with continuous 70 or 8-0 polypropylene (Prolene)monofilament suture. After the procedure, heparin therapy was reversed with protamine sulfate in a 1:1 ratio. The leg, forearm, and chest wounds were closed and the patients were shifted to ICU. Total operation time and number of grafts were recorded.

\section{Technique of Conventional CABG}

After median sternotomy conduits were harvested and prepared and the patients were heparinized with an initial dose of $300 \mathrm{lU} / \mathrm{kg}$ heparin to achieve a target activated clotting time greater than 450 second. CPB was established with ascending aortic and two-stage venous cannulation. The blood pressure was maintained within 50 to $70 \mathrm{~mm} \mathrm{Hg}$. The aorta was cross-clumped, and myocardial protection was achieved with intermittent antegrade cold blood cardioplegia according to a standard protocol. On completion of all the distal anastomoses aortic clamp was released and the proximal anastomoses were performed with partial clamping of the ascending aorta during rewarming. Then the patients were gradually weaned from cardiopulmonary bypass (CPB).Heparin neutralization was done by protamine in 1:1 ratio at the end of the procedure. The leg, forearm, and chest wounds were closed and the patients were shifted to ICU. Total operation time and number of grafts were recorded.

\section{Postoperative Management}

After surgery, all patients were transferred to the intensive care unit (ICU). Cardiac, respiratory, renal function and hourly blood loss were monitored meticulously. Extubation was done as early as possible while the patients fulfill the extubation criteria. Arterial blood gas, serum electrolytes and hematocrit estimation were done as per standard protocol. Haemodynamic and other parameters were managed according to standard protocol. Pre-operative medications continued as per protocol. Patients were encouraged to intensive spirometry and early mobilization. Then the patients were shifted to general wards and subsequently discharged according to unit protocol. Antibiotic administration was continued until the patient hadcentrallines or chest drains (normally 24-48 hours).

\section{Data collection:}

All data were collected from each patient using predesigned questionnaire and collection form. Data were analyzed and verified with statistical program for social sciences (SPSS) using student's t test, fisher's exact test, chi-square test, where appropriate. The descriptive statistics used here were frequency, mean and standard deviation (SD) and compared using student's t test. Categorical data were expressed as percentages and evaluated using Chi-square or Fischer's exact probability test. The level of significant was 0.05 . Any $p$-value $<0.05$ was considered as significant.

\section{Results:}

Patient characteristics are shown in the Table-1. The mean ages of the study sample were61.4 \pm 9.56 years for the OPCAB group and $59.2 \pm 5.87$ years for $C$ CAB group with no significant difference $(p=0.4090 ;>0.05)$ in age distribution. In the study both sexes were homogenously distributed between the two groups but with clear male predominance (90\% in OPCAB vs. $86.7 \%)$. Overweight and obese patients were higher among $C C A B$ group than that in OPCAB group (43.3\% vs. $33.3 \%)$. However, the mean BMI were almost similar $(28.1 \pm 2.38 \mathrm{vs}$. $\left.27.7 \pm 3.4 \mathrm{~kg} / \mathrm{m}^{2}, p=0.955 ;>0.05\right)$. In our study, the commonest co-morbid factor was smoking in both groups (66.7\% in OPCAB vs. $63.3 \%$ in CCAB group). It was followed by dyslipidemia $(63.3 \%$ vs. $53.3 \%)$, hypertension $(53.3 \%$ vs. $50 \%)$ and diabetes mellitus (33.3\% in both groups). Other co-morbid factors were family history of CAD ( $10 \%$ vs. $13.3 \%$ ), past history of CVA (6.7\% vs. $10 \%)$, COPD ( $10 \%$ vs. $10 \%)$, history of MI $(20 \%$ vs. $23.3 \%)$, PVD (10\% vs. $10 \%)$ and renal dysfunction $(6.7 \%$ vs. $3.3 \%)$. All were almost identically distributed between the groups $(p>0.05)$. In the study the mean left ventricular ejection fraction (LVEF) was almost similar in both groups $(39.5 \pm 3.6 \%$ vs. $38.65 \pm 2.7, p=0.1461)$. Preoperative angiographic study demonstrated that majority of the patients had triple vessel disease (TVD) in each group ( $70 \%$ and $83.3 \%$ respectively). The rest had double vessel disease (DVD). The operating time was significantly higher in CCAB group ( $296.3 \pm 34.8 v s$. 268.5 \pm 33.5 minutes, $p<0.001$ ). The mean cardiopulmonary bypass (CPB) time was $110.3 \pm 23.9$ minutes in conventional CABG group. Most of the patients of both groups $(73.3 \%$ and $80 \%$ respectively, $p=0.470)$ required 3 grafts. $2(6.7 \%)$ CCAB and $1(3.3 \%)$ OPCAB patients had 4 distal anastomoses. Among the post-operative variables the mean ventilation time was significantly higher in the CCAB group than those in OPCAB group $(12.3 \pm 0.5$ vs. $8.6 \pm 0.3$ hours, $p<0.001)$. In NICVD there is a trend to use inotropic support to almost all post-CABG patients unless otherwise specified. So, we used 
prolonged post-operative inotropic support ( $>24$ hours) or use of IABP as post-operative outcome variables. In our study $4(6.7 \%)$ CCAB patient required prolonged inotropic support. Among them 2 (3.3\%) required IABP support. On the other hand, one $(1.67 \%)$ of the OPCAB patients received prolonged inotropic support and one $(1.67 \%)$ required IABP.

Total post-operative bleeding was significantly lower in OPCAB (378 $\pm 45 \mathrm{ml}$ vs. $602 \pm 60 \mathrm{ml}, \mathrm{p}<0.001)$ group. Blood product requirement was also significantly lower in OPCAB group $(690 \pm 30$ vs. $1085 \pm 45 \mathrm{ml}, \mathrm{p}<0.0010)$. In this study, the average ICU-stay ( $34.7 \pm 0.9$ hours vs. 43.6 \pm 1.5 hours, $p<0.001)$ and total postoperative hospital stay was also shorter in OPCAB group (8.2 \pm 0.2 days vs.10.3 \pm 0.3 days, $p<0.001)$.

One patient (1.67\%) of OPCAB group and two (3.3\%) of CCAB group $(p=1.000)$ died within 30days of operation. In the immediate postoperative period, $2(3.3 \%)$ of the CCAB patients and $1(1.67 \%)$ of the OPCAB patients developed new $\mathrm{Q}$-wave myocardial infarction (MI). Other patients with post-operative myocardial infarction recovered with conservative management.One patient of CCAB group having LVEF of $30 \%$ required IABP during weaning from $\mathrm{CPB}$. The only death of OPCAB group was due to cardiac temponade from mediastinal bleeding. Re-exploration was done and bleeding was secured. But he died later due to multi-organ dysfunction from prolonged low output syndrome. The other patient of CCAB group died of pulmonary complication (pneumonia) requiring re-intubation. The mean period of mechanical ventilation, amount of blood products needed, length of ICU stay and hospital stay during the early post-operative period- all were significantly greater in CCAB group. Three $(6.7 \%)$ patients of CCAB group and one $(1.67 \%)$ of OPCAB group required re-exploration for bleeding $(p=0.6186)$. Two $(3.3 \%)$ of CCAB patients and one $1(1.67 \%)$ of OPCAB group developed stroke $(p=1.000)$. New postoperative arrhythmias were developed in 12 $(20 \%)$ CCAB and6 (10\%) OPCAB patients $(p=0.236)$. In most (14 patients) of them there were atrial arrhythmia and the remaining (4 patients) had ventricular tachycardia.

LOS (6.7\% vs. $1.67 \%$ ), pulmonary complications (10 vs. $6.7 \%)$, infective complications (10\% vs. $4 \%$ ), and renal dysfunction(10 \% vs. $4 \%$ ), were also more common in CCAB group. Thus, postoperative complications were relatively less common in OPCAB group although statistically not significant. But this might be significant if larger sample would have been taken.

Table-I

Patient characteristics of multivessel coronary artery disease

\begin{tabular}{|c|c|c|c|}
\hline Variables & OPCAB group $(n=60)$ & CCAB group $(n=60)$ & $p$ Value \\
\hline Age, years $\#$ & $61.4 \pm 9.56^{*}$ & $59.2 \pm 5.87^{*}$ & $0.4090^{\text {ns }}$ \\
\hline Male, $\mathrm{n}(\%)^{*}$ & $54(90)$ & $52(86.7)$ & $0.500^{\text {ns }}$ \\
\hline Female, $\mathrm{n}(\%)^{¥}$ & $6(10)$ & $8(13.3)$ & $0.500^{\mathrm{ns}}$ \\
\hline $\operatorname{BMI}\left(\mathrm{kg} / \mathrm{m}^{2}\right)^{\#}$ & $28.1 \pm 2.38^{*}$ & $27.7 \pm 3.4^{*}$ & $0.4568^{\mathrm{ns}}$ \\
\hline Hypertension, $n(\%)^{¥}$ & $32(53.3)$ & $30(50.0)$ & $0.796^{\mathrm{ns}}$ \\
\hline Diabetes mellitus, $\mathrm{n}(\%)^{¥}$ & $20(33.3)$ & $20(33.3)$ & $0.608^{n s}$ \\
\hline Smoking, $\mathrm{n}(\%)^{¥}$ & $40(66.7)$ & $38(63.3)$ & $0.787^{\text {ns }}$ \\
\hline Dyslipidemia, n (\%) & $38(63.3)$ & $32(53.3)$ & $0.432^{\text {ns }}$ \\
\hline Family H/O CAD, n (\%) & $6(10.0)$ & $8(13.3)$ & $0.500^{\mathrm{ns}}$ \\
\hline Past H/O CVA, n (\%) & $4(6.7)$ & $6(10.0)$ & $0.500^{\mathrm{ns}}$ \\
\hline COPD, n $(\%)^{¥}$ & $6(10.0)$ & $6(10.0)$ & $0.665^{\mathrm{ns}}$ \\
\hline History of MI, n (\%) $)^{¥}$ & $12(20.0)$ & $14(23.3)$ & $0.754^{\mathrm{ns}}$ \\
\hline PVD, n $(\%)^{¥}$ & $6(10.0)$ & $6(10.0)$ & $0.665^{\mathrm{ns}}$ \\
\hline Renal dysfunction, $\mathrm{n}(\%)^{\pi}$ & $4(6.7)$ & $2(3.3)$ & $0.500^{\text {ns }}$ \\
\hline Arrhythmia, $n(\%)^{\top}$ & $4(6.7)$ & $4(6.7)$ & $0.694^{\mathrm{ns}}$ \\
\hline LVEF (\%) & $39.5 \pm 3.6^{*}$ & $38.65 \pm 2.7^{*}$ & $0.1461^{\mathrm{ns}}$ \\
\hline NYHA class III or IV, $\mathrm{n}(\%)^{¥}$ & $10(16.67 \%)$ & $8(13.33 \%)$ & $0.952^{\text {ns }}$ \\
\hline CCS angina class III or IV, $\mathrm{n}(\%)^{¥}$ & $26(43.33 \%)$ & $32(53.33)$ & $0.757^{\mathrm{ns}}$ \\
\hline DVD & $18(30.0)$ & $10(16.7)$ & $0.222^{\text {ns }}$ \\
\hline TVD $^{¥}$ & $42(70.0)$ & $50(83.3)$ & $0.222^{\text {ns }}$ \\
\hline
\end{tabular}

*Data are presented as the mean \pm SD for continuous variable.

\# Student's t-Test, $¥$ Chi-square $\left(\zeta^{2}\right)$ Test, ा Fisher’s Exact Test, ns= Non-significant

OPCAB: Off-Pump Coronary Artery Bypass; CCAB: Conventional Coronary Artery Bypass; COPD: Chronic Obstructive Pulmonary Disease; CVA:Cerebrovascular Accident; MI: Myocardial Infarction; NYHA: New York Heart Association; CCS: Canadian Cardiovascular Society Angina Class; PVD: Peripheral Vascular Disease. 
Table-II

Intraoperative Variables

\begin{tabular}{lccc}
\hline Variables & $\begin{array}{c}\text { OPCAB group } \\
(\mathrm{n}=60)\end{array}$ & $\begin{array}{c}\text { CCAB group } \\
(\mathrm{n}=60)\end{array}$ & $\mathrm{p}$ Value \\
\hline $\begin{array}{l}\text { Conversion to CPB, } \mathrm{n}(\%) \\
\text { CPB time, minutes }\end{array}$ & $2(3.33 \%)$ & $110.3 \pm 23.9$ & \\
Total operating time, minutes & & $296.3 \pm 34.8^{*}$ & $<0.0001^{\mathrm{s}}$ \\
Conduit used & $268.5 \pm 33.5^{*}$ & & \\
LIMA, $\mathrm{n}(\%)$ & & $60(100 \%)$ & $0.694 \mathrm{~ns}$ \\
Radial artery, $\mathrm{n}(\%)$ & $60(100 \%)$ & $6(10 \%)$ & $0.500^{\mathrm{ns}}$ \\
SVG, $\mathrm{n}(\%)$ & $8(13.33 \%)$ & $60(100 \%)$ & $0.694^{\mathrm{ns}}$ \\
Graft distribution & $60(100 \%)$ & $60(100 \%)$ & $0.694^{\mathrm{ns}}$ \\
LAD territory, $\mathrm{n}(\%)$ & & $60(100 \%)$ & $0.246 \mathrm{~ns}$ \\
$\quad$ Circumflex territory, $\mathrm{n}(\%)$ & $60(100 \%)$ & $50(83.33 \%)$ & $0.083^{\mathrm{ns}}$ \\
$\quad$ RCA territory, $\mathrm{n}(\%)$ & $56(93.33 \%)$ & $2(3.3 \%)$ & $0.500^{\mathrm{ns}}$ \\
Intraoperative IABPף & $51(85 \%)$ & &
\end{tabular}

* Data are presented as the mean \pm SD for continuous variable.

\# Student's t-Test, $¥$ Chi-square $\left(\chi^{2}\right)$ Test, ๆ Fisher's Exact Test

ns=Non-significant; $s$ = Significant

OPCAB: Off-Pump Coronary Artery Bypass; CCAB: Conventional Coronary Artery Bypass; CPB: Cardiopulmonary Bypass; IABP: Intra-AorticBalloon Pump; LAD: Left Anterior Descending Artery; RCA: Right Coronary Artery; LIMA: Left Internal Mammary Artery; RIMA: Right Internal Mammary Artery; SVG: Saphenous Vein Graft.

Table-III

Comparison of post-operative outcome between groups

\begin{tabular}{|c|c|c|c|}
\hline Variables & $\begin{array}{c}\text { OPCAB group } \\
(n=60)\end{array}$ & $\begin{array}{c}\text { CCAB group } \\
(n=60)\end{array}$ & $\mathrm{p}$ Value \\
\hline 30 days mortality, $\mathrm{n}(\%)^{\top}$ & $1(1.67)$ & $2(3.3)$ & $1.000^{\mathrm{ns}}$ \\
\hline Ventilation time, hours ${ }^{\#}$ & $8.6 \pm 0.3^{*}$ & $12.3 \pm 0.5^{*}$ & $<0.0001^{\mathrm{s}}$ \\
\hline LOS or Prolonged inotropic support" & $1(1.67)$ & $4(6.7)$ & $0.3644^{n s}$ \\
\hline Postoperative IABPף & $0(00)$ & $2(3.3)$ & $0.4958 \mathrm{~ns}$ \\
\hline Total bleeding $(\mathrm{ml})$ \# & $378 \pm 45^{*}$ & $602 \pm 60^{*}$ & $<0.0001^{s}$ \\
\hline Amount of blood products needed $(\mathrm{ml}) \#$ & $690 \pm 30^{*}$ & $1085 \pm 45^{*}$ & $<0.0001^{\mathrm{s}}$ \\
\hline Length of ICU stay(hours) \# & $34.7 \pm 0.9^{*}$ & $43.6 \pm 1.5^{*}$ & $<0.0001^{\mathrm{s}}$ \\
\hline Length of post-operative hospital stay(days) \# & $8.2 \pm 0.2^{*}$ & $10.3 \pm 0.3^{*}$ & $<0.0001^{\mathrm{s}}$ \\
\hline Re-exploration for bleeding ${ }^{\pi}$ & 1(1.67) & $3(5)$ & $0.6186^{\mathrm{ns}}$ \\
\hline Stroke ${ }^{\pi}$ & $1(1.67)$ & $2(3.3)$ & $1.000 \mathrm{~ns}$ \\
\hline Pulmonary complication & $4(6.7)$ & $6(10.0)$ & $0.500^{n s}$ \\
\hline Perioperative MIT & $1(1.67)$ & $2(3.3)$ & $1.000^{n s}$ \\
\hline Arrhythmia $¥$ & $6(10.0)$ & $12(20)$ & $0.236 \mathrm{~ns}$ \\
\hline Surgical site infection $\pi$ & $4(6.7)$ & $6(10.0)$ & $0.500 \mathrm{~ns}$ \\
\hline Renal dysfunction $\pi$ & $4(6.7)$ & $6(10.0)$ & $0.500^{\mathrm{ns}}$ \\
\hline
\end{tabular}

* Data are presented as the mean \pm SD for continuous variable. \# Student’s t-Test, ¥ Chi-square $\left(C^{2}\right)$ Test, ๆ Fisher’s Exact Test ns=Non-significant; $s=$ Significant

LOS= Low Output Syndrome

\section{Discussion:}

In the present study, we analyzed our experience with $O P C A B$ in patients having multivessel disease with reduced left ventricular ejection fraction (d" $50 \%$ ). Although OPCAB approach has fewer short-term complications than conventional $\mathrm{CABG}$, incomplete revascularization is more common with off-pump approach, which led to more complications and repeat revascularization. ${ }^{16}$ Complete revascularization is believed to be important in producing a re-intervention-free result following 
OPCAB. ${ }^{17}$ Meharwal et al. showed the average numbers of grafts were $3.0 \pm 0.7$ for OPCAB group and $3.2 \pm 0.8$ for on-pump group. ${ }^{18}$ Shroyer et al. showed the average numbers of grafts were $2.9 \pm 0.9$ for OPCAB group and $3.0 \pm 1.0$ for on-pump group. ${ }^{19}$ Youn et al. demonstrated in their study that patients with conventional CABG tended to have more grafts, but there was no significant difference in number of distal anastomoses and complete revascularization between the groups. ${ }^{20}$ Technical improvement and experience have led some surgeons to perform off-pump total arterial grafting using two internal thoracic arteries (ITA) or one ITA and radial artery for multivessel coronary artery disease in regular basis. ${ }^{21}$ We have used intracoronary shunts in all patients during distal coronary anastomoses. Positioning and stabilization of the heart in OPCAB, specially during circumflex and posterior descending artery anastomosis, are associated with significant haemodynamic changes. ${ }^{22}$ These changes may be further exacerbated by the snaring of the coronary arteries. Several studies have shown the effectiveness of intracoronary shunts for maintaining myocardial perfusion to avoid ischemia of target vessels during OPCAB, although the use of shunts is not widespread and remains controversial. ${ }^{23} \mathrm{We}$ have found intracoronary shunts useful.

The mean period of mechanical ventilation, amount of blood products needed, length of ICU stay and hospitalstay during the early post-operative period- all were significantly lower in OPCAB group. All these reflect definite clinical advantage as well as favorable economic outcome associated with OPCAB group of patients. Transmission of viral infections, induction of immunologic transfusion reactions, and suppression of the immune system remain important risks related to the transfusion of blood and blood products despite improvements in donor-screening methods. ${ }^{24}$

OPCAB has been shown to be associated with decreased morbidity and mortality in high- risk patients, including the elderly, patients with poor left ventricular function, renal dysfunction, left main stenosis, or chronic obstructive pulmonary disease, and patients with prior neurologic dysfunction. ${ }^{25} \mathrm{As}$ in many studies, our hospital mortalities for OPCAB and CCAB done on patients with multivessel disease with reduced left ventricular ejection fraction (d" $50 \%$ )were comparable. Meharwal et al. reported that the operative mortality was higher in CCAB group (1.86\% vs. $0.97 \%, p<.001) .{ }^{18}$ Ruzzeh et al. in amulti-centre comparative analysis showed similar result $(1.4 \% \text { vs. } 2.9 \%)^{5}$. But, Sajja et al. $(2.8 \%$ vs.3.9\%, $\mathrm{p}=0.746$ ) showed a bit different results. ${ }^{26}$

\section{Study Limitations:}

The present study has several limitations and those are as follows:

1. Sample size was small and patients were selected purposefully.

2. They were not randomly assigned to either group.

3. The surgical procedure either OPCAB or $C C A B$ was determined by the surgeon. Therefore selection bias may affect our findings.

4. The duration of follow up of this study was limited. Clinical outcomes were restricted to 30-days mortality. No data beyond three months follow-up were available. Nothing was mentioned about the quality of life after CABG.

5. As a single institutional study the conclusions may not be applicable in general because of differences in practice patterns of other centres.

6. Other factors such as variations in surgical skill, patient difference in extent or distribution of coronary artery disease and echocardiography reports although unavoidable should also are considered.

\section{Recommendations:}

OPCAB surgery can yield better outcome in terms of shorter recovery time and less adverse effects than CCAB. We recommend OPCAB as a safe and effective surgical strategy for the patients with multivessel coronary artery disease with reduced left ventricular ejection fraction (d"50\%). A prospective large scale multi-institutional randomized trial along with long term follow up and evaluation of graftpatency are necessary to confirm our findings and to define the long term clinical and functional results of both on-pump and off-pump CABG.

Development of well-trained cardiac surgical and anesthetic team, establishment of cardiac surgical centres equipped with modern devices, adequate logistic support and research of ischemic heart disease are needed to ensure up to date service and to widen the future prospects of revascularization procedures.

\section{Acknowledgements:}

I owe my heartfelt gratitude and indebtedness to Professor Dr. Md. Kamrul Hasan, Professor, Department of Cardiac Surgery, NICVD for his active help, guidance and valuable suggestions.

Disclosure of Interests:

I have no potential conflict of interest with respect to the research, authorship, and/or publication of this article. 


\section{References:}

1. Jones RH, Hannan EL, Hammermeister KE. Identification of preoperative variables needed for risk adjustment of short-term mortality after coronary artery bypass graft surgery: The Working Group Panel on the Cooperative CABG Database Project. J AmCollCardiol. 1996;28:1478. 2.

2. Favaloro RG, Effler DB, Groves LK, Shelton WC, Sones FM. Direct myocardial revascularization by saphenous vein graft: Present operative techniques and indications. Ann Thorac Surg. 1970;10:97-111.

3. Wan YP, Arifi A, Wan S, Wong ES, Yip J, Thung KH, et al. Beating heart revascularization with or without cardiopulmonary bypass: Evaluation of inflammatory response in a prospective randomized study. J ThoracCardiovasc Surg. 2004;127:1624-1633.

4. Altarabsheh SE. Outcomes of Off-pump Coronary Artery Bypass Surgery. Bahrain Medical Bulletin. 2009;31:3-12.

5. Ruzzeh SA, Ambler G, Asimakopoulos G,Omar RZ, Hasan R, Fabri A,et al.Off-pump Coronary Artery Bypass (OPCAB) Surgery Reduces Risk-Stratified Morbidity and Mortality: A United Kingdom MultiCentre Comparative Analysis of Early Clinical Outcome. Circulation.2003;108:1-8.

6. Mack MJ, Pfister A, Bachand D, Emery R, Magee MJ, Connolly M, et al.Comparison of coronary artery bypass surgery with and without cardiopulmonary bypass in patients with multivessel disease. J ThoracCardiovasc Surg. 2004;127: 167-173.

7. Arom KV, Flavin TF, Emery RW,Kshettry VR, Janey PA, Petersen RJ, et al.Safety and efficacy of offpumpcoronaryarterybypassgrafting. Ann Thorac Surg.2006;669:704-710.

8. Puskas JD, Wright CE, Ronson RS,Brown WM, Gott JP, GuytonRA, et al. Off-pump multivessel coronary bypass via sternotomy is safe and effective. Ann Thorac Surg. 1998;66:1068-1072.

9. Cohn L. Myocardial Revascularization without Cardiopulmonary Bypass.In:Cohn L, editor. Cardiac Surgery in the Adult. 3rd ed. New York: McGraw Hill Medical; 2008.p. 633-652.

10. Hernandez F, Cohn WE, Baribeau Y. In-Hospital Outcomes of Off-pump Versus On-pump Coronary Artery Bypass Procedure: A Multicenter Experience. Ann Thorac Surg. 2001;72:1528-1534.
11. Roy A, Stanbridge RL, O'Regan D. Progression to $100 \%$ offpump coronary artery bypass with the Octopus-1 dual holder. Heart Surg Forum. 2001;4:174-178.

12. Takai, H., Kobayashi, J., Tagusari, O. Off-pump coronary artery bypass grafting for acute myocardial infarction. Circulation. 2006;70:1303-1306.

13. Fouda M. Coronary artery bypass surgery with onpump beating heart technique. Asian CardiovascThorac Ann. 2007;15:392-5.

14. Ishida M, Kobayashi J, Tagusari O, Bando K, Niwaya $\mathrm{K}$, Nakajima $\mathrm{H}$, et al. Peri-operative advantage of off-pump coronary artery bypass grafting. Circulation. 2002;66:795-799.

15. Royse CF, Royse AG, Wong CT. Assessment of left ventricular function during off-pump coronary artery bypass surgery. Ann ThoracCardiovasc Surg. 2003;19:371-377.

16. Hu S, Zheng Z, Yuan X. Increasing long-term major vascular events and resource consumption in patients receiving off-pump coronary artery bypass grafting: A single-center prospective observational study. Circulation. 2010;121:1800-1808.

17. Gundry SR, Romano MA, Shattuck OH, RazzoukAJ, Bailey LL. Seven-year follow-up of coronary artery bypasses performed with and without cardiopulmonary bypass. J ThoracCardiovasc Surg. 1998;115:1273-1278.

18. Meharwal ZS, Mishra YK, Kohli V,Singh N, Bapna RK, Mehta Y, et al. Multivessel Off-Pump Coronary Artery Bypass: Analysis of 4953 Cases. The Heart Surgery Forum. 2003;6 (3):153-159.

19. Shroyer AL, Grover FL, Hattler B,Collins JF, McDonald GO, Kozora E, et al. On-Pump versus Off-Pump Coronary-Artery Bypass Surgery. N Eng J Med.2009;361(19):1827-1837.

20. Youn YN, Chang BC, Hong YS, Kwak YL, Yoo KJ. Early and mid-term impacts of cardiopulmonary bypass on coronary artery bypass grafting in patients with poor left ventricular dysfunction: A propensity score analysis. Circulation. 2007;71:1387-1394.

21. Raja SG, Siddiqui H, Ilsley CD, Armani M. In-Hospital Outcomes of Off-pump Multivessel Total Arterial and Conventional Coronary Artery Bypass Grafting: Single Surgeon, Single Center Experience. Ann Thorac Surg. 2009;88:47-53. 
22. Mathison M, Edgerton JR, Horswell JL, Akin JJ, Mack MJ. Analysis of hemodynamic changes during beating heart surgical procedures. Ann Thorac Surg.2000;70:1355-1361.

23. Lucchetti V, Capasso F, Caputo M.. Intracoronary shunt prevents left ventricular function impairment during beating heart coronary revascularization. Eur J Cardiothorac Surg.1999;15:255-259.

24. Consten EC, Henny CP, Eijsman L, Dongelmans $\mathrm{DA}$, van Oers $\mathrm{MH}$. The routine use of fresh frozen plasma in operations with cardiopulmonary bypass is not justified. J ThoracCardiovasc Surg. 1996;112:162-167.

25. Boyd WD, Desai ND, Del Rizo DF, Novick R., McKenzi F.N, Menkis AH. Off-pump surgery decreases postoperative complications and resource utilization in the elderly.Ann Thorac Surg. 1999;68:1490 -1493.

26. Sajja LR, Mannam G, Dandu SBR. Off-pump coronary artery bypass grafting in patients with significant left ventricular dysfunction. Ind J Thorac Cadiovasc Surg. 2008;24:110-115. 